\title{
Dopamine receptor D4 promoter hypermethylation increases the risk of drug addiction
}

\author{
HUIHUI JI ${ }^{1 *}$, XUTING XU $^{1 *}$, GUILI LIU ${ }^{1 *}$, HUIFEN LIU ${ }^{2 *}$, QINWEN WANG $^{1}$, WENWEN SHEN ${ }^{2}$, \\ LONGHUI LI $^{2}$, XIAOHU XIE ${ }^{2}$, HAOCHANG HU ${ }^{1}$, LEI XU ${ }^{1}$, WENHUA ZHOU ${ }^{2}$ and SHIWEI DUAN ${ }^{1}$ \\ ${ }^{1}$ Medical Genetics Center, School of Medicine, Ningbo University, Ningbo, Zhejiang 315211; ${ }^{2}$ Laboratory of \\ Behavioral Neuroscience, Ningbo Addiction Research and Treatment Center, Ningbo, Zhejiang 315010, P.R. China
}

Received May 4, 2016; Accepted April 10, 2017

DOI: $10.3892 /$ etm. 2017.5615

\begin{abstract}
Heroin and methylamphetamine (METH) are two addictive drugs that cause serious problems for society. Dopamine receptor D4 (DRD4), a key receptor in the dopaminergic system, may facilitate the development of drug addiction. The aim of the present study was to investigate the association between the promoter methylation level of $D R D 4$ gene and drug addiction. Bisulfite pyrosequencing technology was used to measure the methylation levels of $D R D 4$ promoter in 60 drug addicts and 52 matched controls. Significantly higher levels of DRD4 CpG1 and CpG4 methylation were detected in METH and heroin drug addicts compared with controls $(\mathrm{P}<0.05)$. Male METH addicts exhibited significantly higher DRD4 CpG1, CpG2 and CpG4 methylation levels compared with sex-matched controls $(\mathrm{P}<0.05)$. In heroin addicts, a positive correlation was observed between depression-dejection and DRD4 CpG5 methylation ( $\mathrm{r}=0.537$, $\mathrm{P}=0.039$ ) whereas there was a negative correlation between drug usage frequency and CpG1 methylation ( $\mathrm{r}=-0.632$, $\mathrm{P}=0.011)$. In METH addicts, methylation levels were not significantly associated with depression-dejection and drug usage frequency. In addition, luciferase assays demonstrated that the target sequence of the $D R D 4$ promoter upregulates gene expression. The results of the present study suggest that DNA methylation of DRD4 may be responsible for the pathophysiology of drug addiction.
\end{abstract}

Correspondence to: Professor Shiwei Duan, Medical Genetics Center, School of Medicine, Ningbo University, 818 Fenghua Road, Ningbo, Zhejiang 315211, P.R. China

E-mail: duanshiwei@nbu.edu.cn

Professor Wenhua Zhou, Laboratory of Behavioral Neuroscience, Ningbo Addiction Research and Treatment Center, 42 Northwest Street, Ningbo, Zhejiang 315010, P.R. China

E-mail:whzhou@vip.163.com

${ }^{*}$ Contributed equally

Key words: heroin, methylamphetamine, DNA methylation, DRD4

\section{Introduction}

Drug addiction is a major health and social problem worldwide. The use of heroin (3,6-diacetylmorphine) is associated with the development of drug addiction, even in first-time users, and the symptoms of heroin withdrawal are difficult to endure and often painful $(1,2)$. Long-term use of heroin has severe medical consequences, including scarred veins, bacterial infections of blood vessels, liver and kidney disease and lung complications (3). Methamphetamine (METH) is a novel drug that has increased in popularity over the past few years with an estimated 24 million users worldwide $(4,5)$. It inhibits learning, processing speed and working memory and results in delayed recall (6). It has been reported that METH addiction may also damage dopamine neurons in human brain (7). Heroin was the most commonly used illicit drug in China between 2008 and 2012, followed by METH (8). Intravenous administration is the predominant way in which heroin and METH are used; however, frequent injections coupled with widespread sharing of needles increase the risk of contracting human immunodeficiency virus hepatitis B, C and other blood-borne diseases (3).

Genetic variants have been demonstrated to be involved in the onset $(9)$, development $(10)$ and dependence $(11,12)$ of drug addiction. Furthermore, interactions between genetic and environmental risk factors contribute to the onset and development of drug addiction (13). Environmental risk factors tend to have a greater effect in children with genetic vulnerability (14). As a link between genetics and the environment, epigenetics may serve a crucial role in drug addiction (15).

The dopaminergic pathway, which serves an important role in the reward and reinforcing mechanisms of the brain, may be a target of therapeutic strategies to treat drug addiction $(16,17)$. The dopamine receptor D4 gene (DRD4) encodes the D4 subtype of the dopamine receptor, which belongs to the dopaminergic system (18). The alternative methylation of $D R D 4$ is associated with various mental illnesses (19) and a male-specific association between $D R D 4$ methylation and schizophrenia has been identified (20). It has been reported that the $D R D 4$ promoter is hypermethylated in patients addicted to alcohol (21). However, little is known regarding the exact nature of $D R D 4$ promoter DNA methylation with regards to heroin and METH addiction. 
The aim of the present study was to investigate the association between drug addiction and DRD4 promoter methylation using pyrosequencing in drug addicts and healthy controls.

\section{Materials and methods}

Patients and clinical data collection. A total of 112 participants were recruited from the Ningbo Addiction Research and Treatment Center (Ningbo, China, $\mathrm{n}=60$ ) and Ningbo Blood Bank (Ningbo, China, n=52) from June 2012 to June 2013. A total of 60 drug users, including 30 heroin (mean age, $30.90 \pm 0.97$ years; male: female, 50:50) and $30 \mathrm{METH}$ (mean age, 31.03 \pm 0.99 years; male: female, 50:50) users, were diagnosed by two experienced and professional psychiatrists according to the DSM-IV criteria (22) and the subject who had a history of psychiatric disease, severe disease, and aged less than 18 years or over 65 years was excluded. A total of 52 healthy control subjects (mean age, $30.90 \pm 0.73$ years; male: female, 27:25) were included in the present study; those with any history of drug addiction or nervous system diseases, including mental illness, were excluded. All male patients were interviewed by the questions based on Profile of Mood State (POMS), which was designed to assess transient, distinct mood states (23). The POMS measures six different dimensions of mood, including tension-anxiety, anger-hostility, vigor-activity, fatigue-inertia, depression-dejection, and confusion-bewilderment over a period of time. A five-point scale ranging from 'not at all' to 'extremely' was used to assess the mood tastes of male addicts. Blood samples were gathered from subjects by the same investigators and all samples were stored at $-80^{\circ} \mathrm{C}$ with $1-2 \%$ EDTA-treated tubes. The present study was approved by the Ethics Committees of Ningbo University and Ningbo Addiction Research and Treatment Center. Written informed consent forms were obtained from all subjects.

DNA methylation assay. Genomic DNA was extracted from peripheral blood samples using a nucleic acid extraction kit (\#605003) and a Lab-Aid 820 Automated Blood DNA Extraction system (both from Xiamen Zeesan Biotech Co. lnc., Xiamen, China) and the DNA methylation assay was performed as previously described (24). The following primers were used: Biotin forward, 5'-GGGAGGTTTTGT TAGATATTAGGT-3' and reverse, 5'-CCACCCTAAACC CAATATTTACTCATCTTA-3'; and the sequencing primer, 5'-ACCAAACCAAACCCT-3'. All oligomers were synthesized by Sangon Biotech Co., Ltd. (Shanghai, China).

Construction of recombinant plasmids. Two fragments (DRD4L and DRD4 S) from DRD4 were selected for the present study. DRD4L contained the target sequence whereas DRD4 S did not. The following primers were used for amplification: DRD4L forward, 5'-CTAGCTAGCAGATACCAG GTGGACTAGGGT-3' and reverse, 5'-GGAAGATCTCGG GGAAGGAGAAGAGG-3'; and DRD4 S forward, 5'-CTA GCTAGCTTCAGGGCCTGGTCTGG-3' and reverse, 5'-CTA GCTAGCAGATACCAGGTGGACTAGGGT-3' (Sangon Biotech, Co., Ltd.). A Gel Extraction kit (Omega Bio-Tek, Inc., Norcross, GA, USA) was used to purify polymerase chain reaction products. The insert segments and pGL3 Basic vector (Promega Corporation, Madison, WI, USA) were both digested with NheI and BglII (New England BioLabs, Inc., Ipswich, England), purified using a Cycle Pure kit (Omega, Bio-Tek, Inc.) and the target DNA fragment was cloned into the pGL3 Basic vector using a DNA Ligation kit (Takara Bio, Inc., Otsu, Japan). The pRL-SV40 vector (Promega Corporation), containing the Renilla luciferase gene, was used as a reference.

Cell culture and transfection and reporter gene activity assay. Human HEK293T cells, purchased from the Chinese Academy of Sciences (Shanghai, China) were used in the present study. Cells were cultured in Dulbecco's modified Eagle's medium (DMEM; HyClone; GE Healthcare Life Sciences, Logan, Utah) with $10 \%$ fetal bovine serum (FBS; TransGen Biotech Co., Ltd., Beijing, China) in a humidified $5 \% \mathrm{CO}_{2}$ incubator at $37^{\circ} \mathrm{C}$. When the cells were in exponential growth phase, they were rinsed twice with phosphate-buffered saline (PBS) and plated on 24-well plates at density of $0.5 \times 10^{4} /$ well in $500 \mu \mathrm{l}$ DMEM supplemented with $10 \%$ FBS. Once cells reached $70 \%$ confluence, they were co-transfected with recombinant pGL3 vector and pRL-SV40 using the Lipofectamine ${ }^{\circledR} 2000$ reagent (Invitrogen; Thermo Fisher Scientific, Inc., Waltham, MA, USA) according to the manufacturer's protocol. The medium was replenished 4-6 h following co-transfection. The Renilla and firefly luciferase activities were detected at 475 and $560 \mathrm{~nm}$, respectively, using the SpectraMax 190 microplate reader (Molecular Devices, LLC, Sunnyvale, CA, USA) $24 \mathrm{~h}$ after co-transfection. A dual-luciferase reporter assay system (Promega Corporation) was used to evaluate gene expression levels according to the manufacturer's protocol.

Statistical analysis. Data are presented as mean \pm standard deviation. PASW Statistical 18.0 software (SPSS, Inc., Chicago, IL, USA) was used for all statistical analyses in the present study. Pearson's $\chi^{2}$ test was performed to compare categorical variables and Student's t-test was used to compare the differences of the mean values of continuous variables between the two groups. $\mathrm{P}<0.05$ was considered to indicate a statistically significant difference.

\section{Results}

DNA methylation analysis of the different groups. As presented in Fig. 1, a fragment with five $\mathrm{CpG}$ sites was amplified to evaluate the methylation level of the DRD4 promoter. DRD4 CpG1 and CpG4 methylation levels were significantly higher in all heroin and METH addicts compared with 52 controls (CpG1, P=0.009; and CpG4, P=0.009; Fig. 2A and Table I). A further subgroup analysis of participants addicted to METH or heroin were compared with controls and elevated levels of DRD4 CpG1 and CpG4 methylation were observed in heroin and METH users compared with controls (heroin: CpG1, $\mathrm{P}=0.049$ and $\mathrm{CpG} 4, \mathrm{P}=0.045$; METH: $\mathrm{CpG} 1, \mathrm{P}=0.014$ and CpG4, $\mathrm{P}=0.026$; Fig. $2 \mathrm{~B}$ and $\mathrm{C}$ and Table I).

Correlation analysis between DNA methylation and sex. Sex differences are an important factor for drug addiction (25). Significantly higher DRD4 CpG1 and CpG4 methylation levels 
Table I. Comparisons of dopamine receptor D4 promoter methylation levels among different groups.

\begin{tabular}{|c|c|c|c|c|c|c|}
\hline \multirow[b]{2}{*}{ Groups } & \multirow{2}{*}{$\begin{array}{c}\text { Subgroups } \\
\text { (number ratio) }\end{array}$} & \multicolumn{5}{|c|}{$\mathrm{P}$-value } \\
\hline & & CpG1 & CpG2 & CpG3 & CpG4 & CpG5 \\
\hline \multicolumn{7}{|c|}{ Case vs. control } \\
\hline Total & $(60 / 52)$ & 0.009 & 0.055 & 0.120 & 0.009 & 0.368 \\
\hline Male & $(30 / 27)$ & 0.030 & 0.074 & 0.051 & 0.015 & 0.140 \\
\hline Female & $(30 / 25)$ & 0.162 & 0.410 & 0.935 & 0.257 & 0.992 \\
\hline \multicolumn{7}{|c|}{ Heroin vs. control } \\
\hline Total & $(30 / 52)$ & 0.049 & 0.214 & 0.091 & 0.045 & 0.643 \\
\hline Male & $(15 / 27)$ & 0.233 & 0.464 & 0.202 & 0.173 & 0.837 \\
\hline Female & $(15 / 25)$ & 0.129 & 0.260 & 0.307 & 0.153 & 0.732 \\
\hline \multicolumn{7}{|c|}{ METH vs. control } \\
\hline Total & $(30 / 52)$ & 0.014 & 0.058 & 0.485 & 0.026 & 0.327 \\
\hline Male & $(15 / 27)$ & 0.041 & 0.021 & 0.101 & 0.024 & 0.074 \\
\hline Female & $(15 / 25)$ & 0.431 & 0.253 & 0.352 & 0.709 & 0.731 \\
\hline \multicolumn{7}{|c|}{ Heroin vs. METH } \\
\hline Total & $(30 / 30)$ & 0.658 & 0.686 & 0.322 & 0.982 & 0.611 \\
\hline Male & $(15 / 15)$ & 0.240 & 0.226 & 0.976 & 0.444 & 0.112 \\
\hline Female & $15 / 15)$ & 0.435 & 0.404 & 0.096 & 0.288 & 0.447 \\
\hline
\end{tabular}

METH, methylamphetamine.

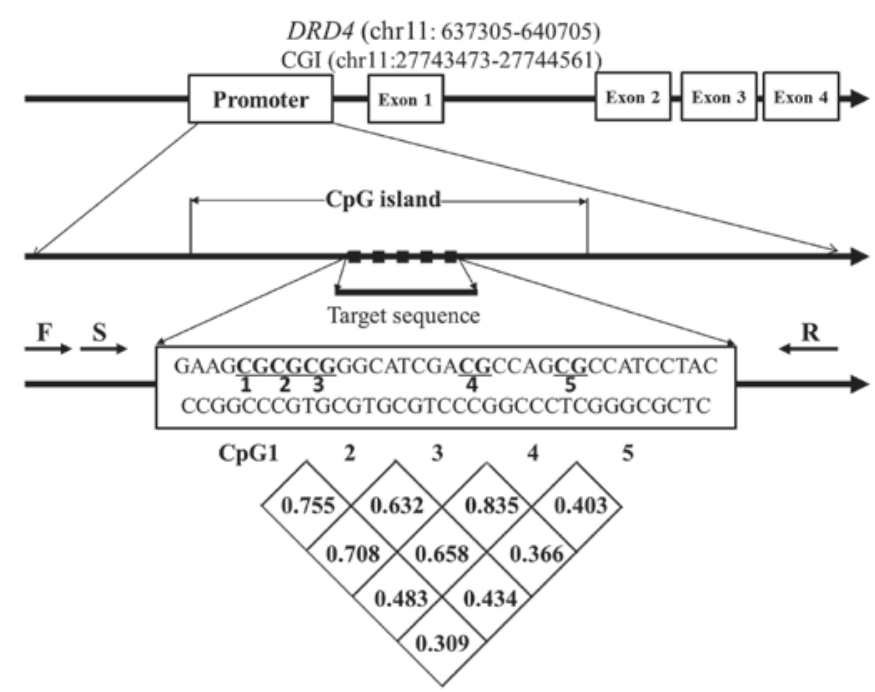

Figure 1. Correlations among five $\mathrm{CpG}$ sites in DRD4 promoter. All of the five $\mathrm{CpG}$ sites are located in the target sequence, which makes DRD4 L different from DRD4 S. CGI, CpG island; F, forward primer; R, reverse primer; S, sequencing primer. DRD4, dopamine receptor D4.

were identified in male heroin addicts and METH addicts compared with healthy male controls (male: CpG1, $\mathrm{P}=0.030$ and $\mathrm{CpG} 4, \mathrm{P}=0.015$; Fig. $2 \mathrm{~A}$ and Table I). Furthermore, levels of DRD4 CpG1, CpG2 and CpG4 methylation were significantly higher in METH male addicts compared with male controls (male: $\mathrm{CpG} 1, \mathrm{P}=0.041$; $\mathrm{CpG} 2, \mathrm{P}=0.021$ and $\mathrm{CpG} 4$, $\mathrm{P}=0.024$; Fig. 2C and Table I). No significant differences in DRD4 methylation levels were observed in male or female heroin addicts compared with sex-matched controls.
Correlation analysis between DNA methylation and phenotypic indices in males. A positive correlation between DRD4 CpG5 methylation level and depression-dejection was observed in male heroin addicts ( $r=0.537, \mathrm{P}=0.039$; Fig. 3 ). Conversely, a negative correlation between DRD4 CpG1 methylation level and usage frequency was identified in heroin addicts ( $\mathrm{r}=-0.632, \mathrm{P}=0.011$; Fig. 3 ). No significant associations were observed between methylation levels and either usage frequency or depression-dejection in METH addicts.

Functional analysis of target fragment. As presented in Fig. 4A, to further validate the function of the target sequence, the two fragments DRD4 L and DRD4 S from the $D R D 4$ promoter were selected to insert into pGL3-basic vectors, respectively. Fluorescence activity was significantly higher in cells transfected with DRD4 L fragment compared with cells transfected with DRD4 S ( $<<0.001$; Fig. 4B). Therefore, the target sequence may have the ability to upregulate the level of the expression of downstream genes.

\section{Discussion}

In the present study, the levels of DRD4 methylation in drug addicts and matched controls were evaluated to determine the contribution of $D R D 4$ promoter methylation to drug addiction. DRD4 CpG1 and CpG4 methylation levels were significantly higher in cases, including heroin and METH, compared with controls. Significantly elevated levels of DRD4 CpG1 and CpG4 methylation were also observed in heroin and METH users compared with controls, separately. There were significantly higher levels of DRD4 CpG1 and CpG4 methylation in 

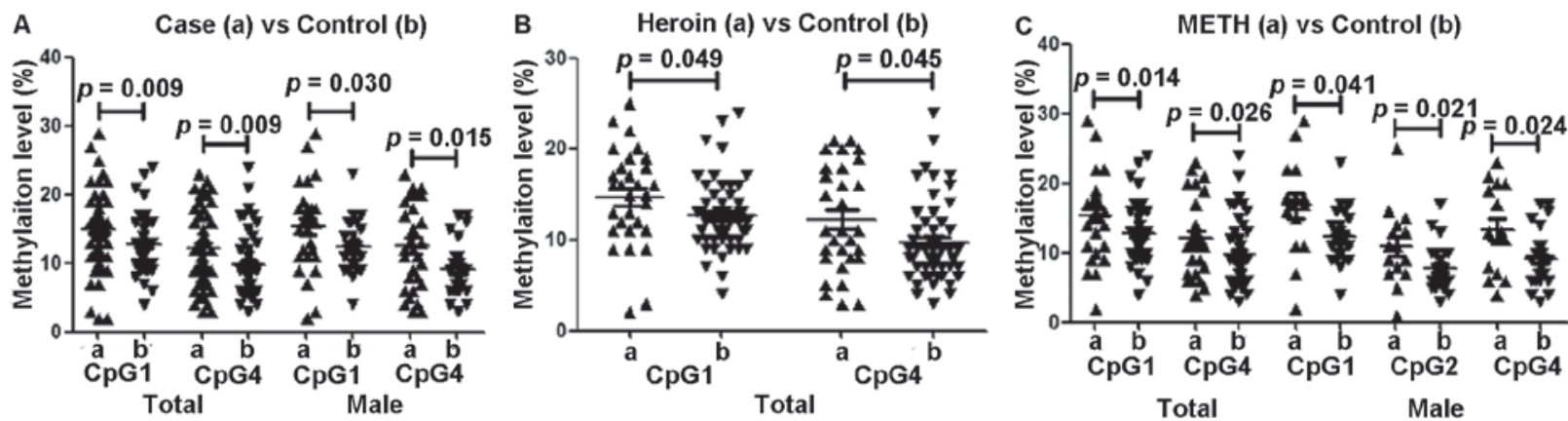

Figure 2. Differences in DRD4 promoter methylation levels among different subgroups. Methylation levels of CpG1 and CpG4 were indicated in (A) METH and heroin cases. Total group includes heroin addicts and METH addicts and healthy controls. Male group includes total male addicts and male healthy controls. (B) Heroin cases alone compared with respective controls. Total group includes heroin addicts and healthy controls. (C) METH cases alone compared with respective controls. Total group includes METH addicts and healthy controls. Male group includes male METH addicts and male healthy controls DRD4, dopamine receptor D4; METH, methylamphetamine; a, METH cases; b, controls.
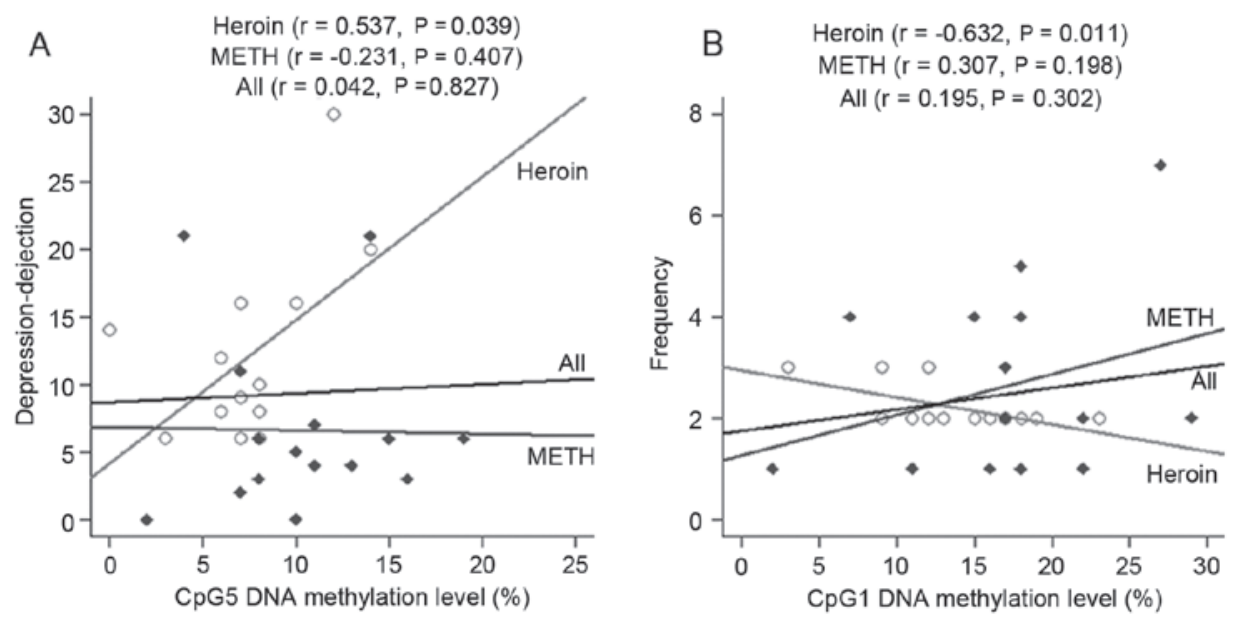

Figure 3. Correlation between DRD4 methylation levels and phenotypes. Correlation analysis of CpG5 DNA methylation levels with (A) depression-dejection and (B) usage frequency in heroin and METH addicts. Circles and squares stand indicate methylation levels of DRD4 promoter in heroin and METH addicts, respectively. DRD4, dopamine receptor D4; METH, methylamphetamine.

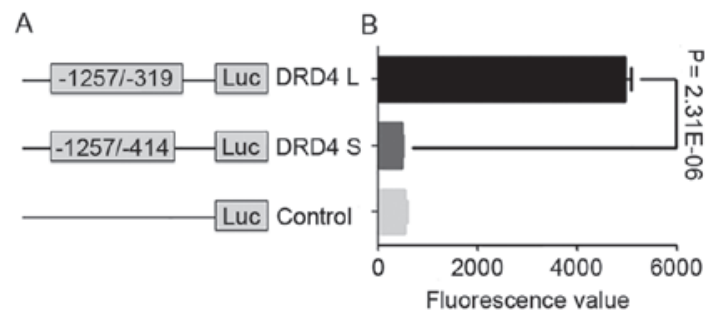

Figure 4. Luciferase assay of the pyrosequenced fragment in the DRD4 promoter. (A) Locations of DRD4 L fragment and DRD4 S fragment in the DRD4 gene. DRD4 L indicates the fragment containing the pyrosequenced sequence. DRD4 S indicates the fragment did not contain that sequence. (B) Fluorescence intensities in control, DRD4 S and DRD L.

male heroin and METH addicts compared with male healthy controls. Furthermore, significantly higher methylation levels of $\mathrm{CpG} 1, \mathrm{CpG} 2$ and $\mathrm{CpG} 4$ were detected in METH male addicts compared with male healthy controls. In addition, in heroin addicts, a positive correlation between CpG1 methylation and depression-dejection was observed, whereas a negative correlation between $\mathrm{CpG1}$ methylation and usage frequency was identified. Gene promoter methylation level was reversely associated to its expression (26). The subsequent transfection experiment also suggests that the targeted sequence was able to upregulate levels of $D R D 4$ gene expression.

Drug addiction is a serious problem in many countries, including China. The dopaminergic system serves a crucial role in the reward and reinforcement mechanisms of the brain (16). DRD4 is an important member of the dopaminergic system and the association between the DRD4 gene and heroin has been reported previously (27). A previous case-control study revealed that a promoter polymorphism (rs936462) of $D R D 4$ was indirectly associated with heroin addiction via the -521 C/T (rs1800955) polymorphism in promoter (11). DNA methylation is involved in the long-term memory formation (28) and serves a crucial role in a number of mental disorders, including those associated with drug addiction (29). The present study demonstrated that significantly increased $\mathrm{CpG} 1$ and $\mathrm{CpG} 4$ methylation levels were identified in heroin addicts compared with controls. According to subsequent functional experiments, the target sequence measured in the present study was able to upregulate the expression of downstream genes. The results of the present study indicate that DNA methylation alteration in $D R D 4$ may be associated with heroin addiction. 
Similar to heroin addiction, the etiology of METH abuse is complex. The association between DRD4 and METH abuse was determined by a previous study (30). In the present study, increased methylation levels of $D R D 4$ promoter were detected in METH addicts compared with normal controls. Furthermore, significantly higher levels of DRD4 were identified in male METH addicts following subgroup analysis by sex. The sex of addicts may affect METH addiction. The dopaminergic system is sexually dimorphic and the physiological status and influence of drug abuse of the dopamine system differ between males and females (31). Thus, DNA methylation may serve different roles in males and females; however, the association between DNA methylation and sex requires further study.

Heroin addicts have lower levels of striatal dopamine transporter and more depressive symptoms compared with healthy controls $(32,33)$. A previous study on genome-wide DNA methylation profiling identified that DNA methylation levels were lower in patients with major depressive disorder compared with controls and that the majority of the methylated sites $(85.7 \%)$ were located on the promoter $\mathrm{CpG}$ islands (34). Additionally, a previous study suggested that maternal depression is associated with DNA methylation changes in cord blood T lymphocytes and adult hippocampi (35). In the present study, a positive correlation between CpG5 methylation and depression-dejection was detected in heroin addicts, indicating that the DRD4 CpG5 methylation level may contribute to the onset of depression in heroin addicts. Depressive symptoms in addicts may be associated with heroin withdrawal, which compared with withdrawal from other opiates, is considered to be more uncomfortable, difficult and painful $(1,2)$. By contrast, a negative correlation was identified between $\mathrm{CpG} 1$ methylation and usage frequency in heroin addicts. These observations suggest that the frequency of heroin usage may alter CpG1 methylation.

Although the present study identified a number of meaningful results, there were a number of limitations. Firstly, the sample size was relatively small for the associated study. Secondly, a fragment that contains only five $\mathrm{CpG}$ sites in the $D R D 4$ promoter was tested in the present study and this fragment may not be sufficient to reflect the DNA methylation state of the whole gene. Thirdly, tissue specificity between the peripheral blood and brain still needs to be taken into account, although a strong correlation of gene methylation between the blood and brain has previously been observed (36). Lastly, a functional study of the target sequence on the RNA and the protein expression levels was not performed due to a lack of samples. Further studies investigating the mechanisms regulated by the target sequence are required.

In conclusion, the present study demonstrates that DRD4 promoter hypermethylation may be associated with heroin and METH addiction. The results suggest that changes in DRD4 promoter methylation may affect $D R D 4$ expression and the behavior of drug addicts.

\section{Acknowledgements}

The present work was supported by the National Basic Research Program of China (grant no. 2015CB553504), Nature Science Foundation of China (grant nos. U1132602, 81171257, 81371469 and 81471398), Natural Science Foundation of Zhejiang
Province (grant nos. LR13H020003, Y15H090032 and LY14H310002), K.C. Wong Magna Fund in Ningbo University, Public Technology Research and Social Development Project of Zhejiang Province and Ningbo (grant nos. 2015C33155 and 2013C50033) and Excellent Paper Cultivated Fund of Ningbo University (grant no. 014-F01660148000).

\section{References}

1. Hubner CB and Kornetsky C: Heroin, 6-acetylmorphine and morphine effects on threshold for rewarding and aversive brain stimulation. J Pharmacol Exp Ther 260: 562-567, 1992.

2. Oppenheimer E, Tobutt C, Taylor C and Andrew T: Death and survival in a cohort of heroin addicts from London clinics: A 22-year follow-up study. Addiction 89: 1299-1308, 1994.

3. Hosztafi S: Heroin addiction. Acta Pharm Hung 81: 173-183, 2011 (In Hungarian).

4. Huang K, Zhang L and Liu J: Drug problems in contemporary China: A profile of Chinese drug users in a metropolitan area. Int J Drug Policy 22: 128-132, 2011.

5. Results from the 2013 National Survey on Drug Use and Health: Summary of National Findings. Journal, 2014.

6. Lundqvist T: Cognitive consequences of cannabis use: Comparison with abuse of stimulants and heroin with regard to attention, memory and executive functions. Pharmacol Biochem Behav 81: 319-330, 2005.

7. Kish SJ, Boileau I, Callaghan RC and Tong J: Brain dopamine neurone 'damage': Methamphetamine users vs. Parkinson's disease-a critical assessment of the evidence. Eur J Neurosci 45: 58-66, 2017.

8. Tettey J, Wong YL, Levissianos S and Eichinger N: Patterns and trends of amphetamine-type stimulants (ATS) and other drugs-challenges for asia and the pacific. J, 2013.

9. Li T, Yu S, Du J, Chen H, Jiang H, Xu K, Fu Y, Wang D and Zhao M: Role of novelty seeking personality traits as mediator of the association between COMT and onset age of drug use in Chinese heroin dependent patients. PLoS One 6: e22923, 2011.

10. Xie X, Xu L, Liu H, Chen W, Zhuang D, Zhang J, Duan S and Zhou W: Positive association between-1021TT genotype of dopamine beta hydroxylase gene and progressive behavior of injection heroin users. Neurosci Lett 541: 258-262, 2013.

11. Vereczkei A, Demetrovics Z, Szekely A, Sarkozy P, Antal P, Szilagyi A, Sasvari-Szekely $M$ and Barta C: Multivariate analysis of dopaminergic gene variants as risk factors of heroin dependence. PLoS One 8: e66592, 2013.

12. Peng S, Du J, Jiang H, Fu Y, Chen H, Sun H, Wang D, Yu S and Zhao M: The dopamine receptor D1 gene is associated with the length of interval between first heroin use and onset of dependence in Chinese Han heroin addicts. J Neural Transm (Vienna) 120: 1591-1598, 2013.

13. In: Pathways of addiction: Opportunities in drug abuse research. Washington (DC), 1996.

14. Maslowsky J, Schulenberg JE and Zucker RA: Influence of conduct problems and depressive symptomatology on adolescent substance use: Developmentally proximal versus distal effects. Dev Psychol 50: 1179-1189, 2014.

15. Feng $\mathrm{J}$ and Nestler EJ: Epigenetic mechanisms of drug addiction. Curr Opin Neurobiol 23: 521-528, 2013.

16. Everitt BJ, Parkinson JA, Olmstead MC, Arroyo M, Robledo P and Robbins TW: Associative processes in addiction and reward. The role of amygdala-ventral striatal subsystems. Ann N Y Acad Sci 877: 412-438, 1999.

17. Di Ciano P, Grandy DK and Le Foll B: Dopamine D4 receptors in psychostimulant addiction. Adv Pharmacol 69: 301-321, 2014.

18. Kirley A, Hawi Z, Daly G, McCarron M, Mullins C, Millar N, Waldman I, Fitzgerald $M$ and Gill M: Dopaminergic system genes in ADHD: Toward a biological hypothesis. Neuropsychopharmacology 27: 607-619, 2002.

19. Sharma A, Kramer ML, Wick PF, Liu D, Chari S, Shim S, Tan W, Ouellette D, Nagata M, DuRand CJ, et al: D4 dopamine receptor-mediated phospholipid methylation and its implications for mental illnesses such as schizophrenia. Mol Psychiatry 4: 235-246, 1999.

20. Cheng J, Wang Y, Zhou K, Wang L, Li J, Zhuang Q, Xu X, Xu L, Zhang K, Dai D, et al: Male-specific association between dopamine receptor D4 gene methylation and schizophrenia. PLoS One 9: e89128, 2014. 
21. Zhang H,Herman AI, Kranzler HR, Anton RF, Zhao H, Zheng W and Gelernter J: Array-based profiling of DNA methylation changes associated with alcohol dependence. Alcohol Clin Exp Res 37 (Suppl 1): E108-E115, 2013.

22. Hasin DS, O'Brien CP, Auriacombe M, Borges G, Bucholz K, Budney A, Compton WM, Crowley T, Ling W, Petry NM, et al: DSM-5 criteria for substance use disorders: Recommendations and rationale. Am J Psychiatry 170: 834-851, 2013.

23. Shen W, Liu Y, Li L, Zhang Y and Zhou W: Negative moods correlate with craving in female methamphetamine users enrolled in compulsory detoxification. Subst Abuse Treat Prev Policy 7: 44, 2012.

24. Chang L, Wang Y, Ji H, Dai D, Xu X, Jiang D, Hong Q, Ye H, Zhang X, Zhou X, et al: Elevation of peripheral BDNF promoter methylation links to the risk of Alzheimer's disease. PLoS One 9: e110773, 2014

25. Berry A, Raggi C, Borgi M and Cirulli F: Sex-driven vulnerability in stress and drug abuse. Ann Ist Super Sanita 52: 167-175, 2016.

26. Li S, Zhu Y, Zhi L, Han X, Shen J, Liu Y, Yao J and Yang X: DNA methylation variation trends during the embryonic development of chicken. PLoS One 11: e0159230, 2016.

27. Lai JH, Zhu YS, Huo ZH, Sun RF, Yu B, Wang YP, Chai ZQ and Li SB: Association study of polymorphisms in the promoter region of DRD4 with schizophrenia, depression, and heroin addiction. Brain Res 1359: 227-232, 2010.

28. Miller CA and Sweatt JD: Covalent modification of DNA regulates memory formation. Neuron 53: 857-869, 2007.

29. Tsankova N, Renthal W, Kumar A and Nestler EJ: Epigenetic regulation in psychiatric disorders. Nat Rev Neurosci 8: 355-367, 2007.
30. Bousman CA, Glatt SJ, Everall IP and Tsuang MT: Genetic association studies of methamphetamine use disorders: A systematic review and synthesis. Am J Med Genet B Neuropsychiatr Genet 150B: 1025-1049, 2009.

31. Mendrek A: Are there any sex/gender differences in drug use and drug addiction?. Sante Ment Que 39: 57-74, 2014 (In French).

32. Sarchiapone M, Carli V, Camardese G, Cuomo C, Di Giuda D, Calcagni ML, Focacci C and De Risio S: Dopamine transporter binding in depressed patients with anhedonia. Psychiatry Res 147: 243-248, 2006.

33. Roselli F, Pisciotta NM, Perneczky R, Pennelli M, Aniello MS, De Caro MF, Ferrannini E, Tartaglione B, Defazio G, Rubini G and Livrea P: Severity of neuropsychiatric symptoms and dopamine transporter levels in dementia with Lewy bodies: A 123I-FP-CIT SPECT study. Mov Disord 24: 2097-2103, 2009.

34. Numata S, Ishii K, Tajima A, Iga J, Kinoshita M, Watanabe S, Umehara H, Fuchikami M, Okada S, Boku S, et al: Blood diagnostic biomarkers for major depressive disorder using multiplex DNA methylation profiles: Discovery and validation. Epigenetics 10: 135-141, 2015.

35. Nemoda Z, Massart R, Suderman M, Hallett M, Li T, Coote M, Cody N, Sun ZS, Soares CN, Turecki G, et al: Maternal depression is associated with DNA methylation changes in cord blood T lymphocytes and adult hippocampi. Transl Psychiatry 5: e545, 2015.

36. Jiang D, Zheng D, Wang L, Huang Y, Liu H, Xu L, Liao Q, Liu P, Shi X, Wang Z, et al: Elevated PLA2G7 gene promoter methylation as a gender-specific marker of aging increases the risk of coronary heart disease in females. PLoS One 8: e59752, 2013. 